\title{
Response to correspondence from the ESSA Statement authors
}

\author{
Kathleen A. Martin Ginis $\mathbb{D}^{1} \cdot$ Jan W. van der Scheer ${ }^{2} \cdot$ Amy E. Latimer-Cheung $^{3} \cdot$ Andy Barrow $^{4} \cdot$ Chris Bourne $^{5} \cdot$ \\ Peter Carruthers ${ }^{6} \cdot$ Marco Bernardi $^{7} \cdot$ David S. Ditor $^{8} \cdot$ Sonja Gaudet ${ }^{9} \cdot$ Sonja de Groot ${ }^{10} \cdot$ Keith C. Hayes $^{11}$. \\ Audrey L. Hicks ${ }^{12}$. Christof A. Leicht ${ }^{2} \cdot$ Jan Lexell ${ }^{13}$ - Steven Macaluso ${ }^{14} \cdot$ Patricia J. Manns $^{15}$. \\ Christopher B. McBride ${ }^{9}$. Vanessa Noonan ${ }^{16}$ - Pierre Pomerleau ${ }^{17}$ - James H. Rimmer ${ }^{18} \cdot$ Robert B. Shaw $^{19}$. \\ Brett Smith $^{20} \cdot$ Karen M. Smith ${ }^{3} \cdot$ John Steeves $^{21} \cdot$ Dot Tussler $^{22} \cdot$ Christopher R. West $^{1} \cdot$ Dalton L. Wolfe ${ }^{23}$. \\ Victoria L. Goosey-Tolfrey²
}

Received: 10 December 2017 / Revised: 10 December 2017 / Accepted: 13 December 2017 / Published online: 18 January 2018

(c) International Spinal Cord Society 2018

We thank the ESSA statement authors for their correspondence regarding the International Scientific SCI Exercise Guidelines (ISSEG) [1]. We believe issues raised in their correspondence reflect differences in our two groups' philosophical, practical, scientific, and methodological orientations toward spinal cord injury (SCI) exercise guidelines.

We believe guideline development should meaningfully engage the people who use the guidelines.

People living with SCI, SCI organizations, physiatrists, physiotherapists, and other stakeholders participated in the ISSEG guideline development process. Their voices were loud and clear: they do not have confidence in the World Health Organization's (WHO) [2] and ESSA's $150 \mathrm{~min}$ per week guideline [3] because it is neither based on SCI-specific evidence nor feasible. Stakeholders want SCIspecific guidelines [1]. The ISSEG reflect the concerns, values, and preferences of the SCI community. The ESSA statement does not.

We believe SCI exercise guidelines should be developed with the same rigor as guidelines for the general population.

The ISSEG were developed through a rigorous, systematic, and transparent process (i.e., AGREE [4]) that fully adheres to internationally accepted standards for formulating clinical practice and physical activity (PA) guidelines for the general population (e.g.,WHO) [2]. The ESSA statement was not formulated using AGREE and its authors acknowledge their "exercise recommendations....are somewhat arbitrary" (p. 112) [3].

Kathleen A. Martin Ginis

kathleen_martin.ginis@ubc.ca

Extended author information available on the last page of the article
We believe SCI exercise guidelines should be developed by considering all relevant SCI exercise evidence.

The ISSEG are underpinned by a systematic review of 211 SCI studies [5]. Evidence for the effects of exercise, specific exercise prescriptions, representativeness of study participants, and adverse events were synthesized and appraised. The ESSA authors are incorrect in stating the fitness guideline is based on six studies. While the six highest quality (i.e., Levels 1-2) studies provided the guideline's foundation, evidence from 29 Levels 3-4 studies supported its effectiveness and safety. Meanwhile, the ESSA statement "is based on the dose-response relationship between physical activity and disease risk in the general population" (p. 111) [3], and is merely an endorsement of the WHO's generic PA guideline [2].

We would also like to address some specific points raised in the ESSA authors' correspondence:

"Implying that sub-threshold volumes will not confer health/fitness benefits."

The ISSEG preamble states: "doing exercise below the recommended levels may or may not bring small changes in fitness or cardiometabolic health." Our language is deliberate. No Levels 1,2 , 3, or 4 SCI study of aerobic exercise, or aerobic plus strength exercise, has produced significant fitness or cardiometabolic health (CMH) benefits with $<20 \mathrm{~min}$ moderate-vigorous intensity aerobic exercise $2 \times$ per week, or $<2$ bouts of strength exercise per week [5]. We recognize, however, there may be shorter, effective exercise protocols not yet scientifically documented (e.g., high-intensity interval training protocols).

Concerns with stating a "minimum"level of activity.

The authors imply that the evidence base demonstrates a "given-dose effect" rather than a "minimum-dose effect". 
Indeed, no study has directly addressed the exercise doseresponse issue in people with SCI. However, as explained above, a minimum amount is needed to improve fitness. Regarding $\mathrm{CMH}$, the three highest quality studies (Level 2) showed positive effects on $\mathrm{CMH}$ indices using an exercise intervention of $3 \times 30-44$-min sessions per week of $\geq$ moderate intensity [5]. Without evidence for a "lower" minimum and given need for SCI-specific guidelines to improve $\mathrm{CMH}$, we are comfortable endorsing $3 \times 30$-min sessions per week of $\geq$ moderate intensity exercise as a minimum to improve CMH [1]. Furthermore, stating a minimum target is important for PA surveillance and goal setting, and is characteristic of virtually all PA guidelines (e.g., WHO [2]).

We will "cause a relative overestimation of the number of people with SCI who are sufficiently active for good health compared with the general population."

In Canada, about $44 \%$ of non-disabled adults report sufficient activity for good health [6]. A recent Canadian study of 73 adults with SCI showed $12 \%$ reported moderate-vigorous aerobic activity $\geq 2 \times$ per week for $\geq 20$ min plus $\geq 2 \times$ per week strength exercise [7] (i.e., ISSEG fitness guideline [1]). As $60 \%$ of Canadian adults with SCI report no moderate-vigorous exercise whatsoever (secondary analysis of Martin Ginis et al. [8]), even with a "lower" guideline, we are far from SCI "sufficiently active" rates approximating those of the general population.

We are "creating the impression that people with SCI do not need to be as physically active as the general population in order to be healthy."

"Healthy" has myriad meanings. We assume the authors mean CMH (encompassing "traditional" and "non-traditional" indices). The available evidence suggests people with SCI can improve CMH with a "lower" exercise dose than able-bodied individuals [5]. The three Level 2 studies showed improvements in the reviewed $\mathrm{CMH}$ indices with exercise $3 \times$ per week for $30-44$ min $\geq$ moderate intensity [5]. Evidence from eight Levels 3-4 studies supported these results [5].

The ESSA statement authors conclude their correspondence by advising readers to critically evaluate the primary evidence underpinning the guidelines. We agree wholeheartedly and, as such, have made all of our evidence summary tables available [1]. We also encourage readers to consider the altered cardiovascular disease-risk profile, altered response to exercise [9], and extensive PA barriers experienced by people with SCI and to decide which is the better recommendation: exercise guidelines merely lifted from the general population [3] or rigorously developed SCI guidelines underpinned by SCI-specific evidence [1].

Conflict of interest The authors declare that they have no conflict of interest.

\section{References}

1. Martin Ginis KA, van der Scheer JW, Latimer-Cheung AE, Barrow A, Bourne C, Carruthers P, et al. Evidence-based scientific exercise guidelines for adults with spinal cord injury: an update and a new guideline. Spinal Cord. 2017. https://doi.org/10.1038/ s41393-017-0017-3.

2. WHO. Global recommendations on physical activity for health. 2010. http://www.who.int/dietphysicalactivity/factsheet_ recommendations/en/. Accessed 10 Dec 2017.

3. Tweedy SM, Beckman EM, Geraghty TJ, Theisen D, Perret C, Harvey LA, et al. Exercise and Sports Science Australia (ESSA) position statement on exercise and spinal cord injury. J Sci Med Sport. 2017;20:108-15.

4. Brouwers MC, Kho ME, Browman GP, Burgers JS, Cluzeau F, Feder G, et al. AGREE II: advancing guideline development, reporting and evaluation in health care. Can Med Assoc J. 2010;182:E839-E842.

5. van der Scheer JW, Martin Ginis KA, Ditor DS, Goosey-Tolfrey V, Hicks AL, West CR, et al. Effects of exercise on fitness and health of adults with spinal cord injury: a systematic review. Neurology. 2017;89:1-10.

6. Statistics Canada. A comparison of self-reported leisure-time physical activity and measured moderate-to-vigorous physical activity in adolescents and adults. 2014. http://www.statcan.gc.ca/ pub/82-003-x/2014007/article/14038-eng.htm. Accessed 10 Dec 2017.

7. Rocchi M, Routhier F, Latimer-Cheung AE, Martin Ginis KA, Noreau L, Sweet SN. Are adults with spinal cord injury meeting the spinal cord injury-specific physical activity guidelines? A look at a sample from a Canadian province. Spinal Cord. 2017;5:454-9.

8. Martin Ginis KA, Latimer AE, Arbour-Nicitopoulos KP, Buchholz AC, Bray SR, Craven BC, et al. Leisure time physical activity in a population-based sample of people with spinal cord injury Part I: demographic and injury-related correlates. Arch Phys Med Rehabil. 2010;91:722-8.

9. Nash MS. Exercise as a health-promoting activity following spinal cord injury. J Neurol Phys Ther. 2005;29:87-103.

\section{Affiliations}

Kathleen A. Martin Ginis $\mathbb{D}^{1} \cdot$ Jan W. van der Scheer ${ }^{2} \cdot$ Amy E. Latimer-Cheung $^{3} \cdot$ Andy Barrow $^{4} \cdot$ Chris Bourne $^{5}$.

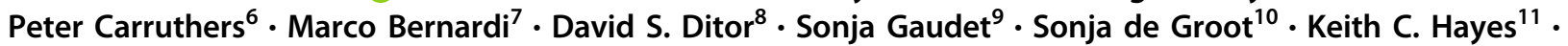
Audrey L. Hicks ${ }^{12}$. Christof A. Leicht ${ }^{2}$. Jan Lexell ${ }^{13}$ - Steven Macaluso ${ }^{14} \cdot$ Patricia J. Manns $^{15}$. Christopher B. McBride ${ }^{9} \cdot$ Vanessa Noonan $^{16} \cdot$ Pierre Pomerleau $^{17} \cdot$ James H. Rimmer $^{18} \cdot$ Robert B. Shaw $^{19}$. 
Brett Smith ${ }^{20} \cdot$ Karen M. Smith ${ }^{3} \cdot$ John Steeves ${ }^{21} \cdot$ Dot Tussler $^{22} \cdot$ Christopher R. West $^{1} \cdot$ Dalton L. Wolfe ${ }^{23}$. Victoria L. Goosey-Tolfrey ${ }^{2}$

1 University of British Columbia, ICORD, Vancouver, Canada

2 Loughborough University, Loughborough, UK

3 Queen's University, Kingston, Canada

4 Paralympian and Inspirational Speaker, London, UK

5 Spinal Cord Injury, Ontario, Canada

6 Bromakin Wheelchairs, Loughborough, UK

7 Sapienza University of Rome, Rome, Italy

8 Brock University, Ontario, Canada

9 Spinal Cord Injury BC, Vancouver, Canada

10 University of Groningen, Groningen, The Netherlands

11 Ontario Neurotrauma Foundation, Ontario, Canada

12 McMaster University, Ontario, Canada

13 Lund University, Lund, Sweden
14 Western University, Ontario, Canada

15 University of Alberta, Alberta, Canada

16 Rick Hansen Institute, Vancouver, Canada

17 Institut de Réadaptation en Déficience Physique de Québec, Ville de Québec, Canada

18 University of Alabama at Birmingham, Alabama, USA

19 University of British Columbia, British Columbia, Canada

20 University of Birmingham, Birmingham, UK

21 ICORD University of British Columbia, Vancouver, Canada

22 National Spinal Injuries Centre, Stoke Mandeville Hospital, Aylesbury, UK

23 Parkwood Institute, Lawson Health Research Institute, Ontario, Canada 